\title{
Cryogenically-cooled Ho:YAG laser in-band pumped by a Tm fibre laser
}

\author{
J. W. Kim ${ }^{1}$, J. I. Mackenzie ${ }^{1}$, W.O.S. Bailey ${ }^{2}$, L. Pearson ${ }^{1}$, D. Y. Shen ${ }^{1}$, Y. Yang ${ }^{2}$, and W.A. Clarkson ${ }^{1}$ \\ 1 Optoelectronics Research Centre, University of Southampton, Highfield, Southampton SO17 1BJ, United Kingdom. \\ 2 School of Engineering Sciences, University of Southampton, Highfield, Southampton SO17 1BJ, United Kingdom
}

Scaling of laser power and brightness to meet the needs of ever-demanding applications is a demanding task which continues to preoccupy many within the laser community. In conventional 'bulk' solid-state lasers the main obstacle is heat generation in the laser medium and its associated detrimental effects. Methods for combating these problems have been the focus of much research, resulting in many novel laser geometries with improved thermal management and reduced thermal lensing, but often at the expense of increased complexity and reduced flexibility. An alternative approach, which is beginning to attract a great deal of interest, is to operate the laser with the laser medium maintained at cryogenic temperatures $(\sim 77 \mathrm{~K})$, where the effects of heat loading are dramatically reduced due to a large increase in thermal conductivity and a large decrease in the temperature coefficient of refractive index (dn/dT) and expansion coefficient [1]. In host materials such as YAG the net reduction in thermal effects can be over 50 times compared to operation at room temperature. In the case of diode-pumped Yb:YAG lasers, the combined effect of a massive reduction in thermo-optic aberrations and lower re-absorption loss has allowed very impressive results to be achieved in terms of output power and beam quality from relatively simple laser resonator configurations [2]. In this paper we report on preliminary work ultimately aimed at achieving a further reduction in thermal effects by combining the advantages of cryogenic cooling with a very low quantum defect fibre-laser-pumping of bulk solid-state lasers. Here we describe preliminary results for a cryogenically-cooled Ho:YAG laser in-band pumped by a high-power Tm-doped silica fibre laser.

The Tm fibre pump laser was constructed in-house from a Tm-doped polarization-maintaining (PM) doubleclad fibre with a core of diameter $25 \mu \mathrm{m}(0.1 \mathrm{NA})$ surrounded by a silica inner-cladding with a diameter of 400 $\mu \mathrm{m}$. Pump light was provided by two spatially-combined diode-stacks with a combined output power of $240 \mathrm{~W}$ at $795 \mathrm{~nm}$. The pump beam was split into two, of roughly equal powers, using a knife-edged mirror and was launched into both ends of the fibre with an efficiency of $>83 \%$. The effective absorption coefficient for pump light at $795 \mathrm{~nm}$ in this fibre was measured to be $\sim 4.5 \mathrm{~dB} / \mathrm{m}$, and hence a fibre of $\sim 3.5 \mathrm{~m}$ length was used for our experiment. The fibre laser cavity was formed by the $\sim 4 \%$ Fresnel reflection from the perpendicularly polished fibre facet at the output end and, at the opposite end, by an external cavity with a diffraction grating for wavelength tuning. In this experiment the Tm fibre laser wavelength was tuned to the absorption peak at 1932 $\mathrm{nm}$ in Ho :YAG. At this operating wavelength we could obtain over $100 \mathrm{~W}$ of output with a slope efficiency of $56 \%$ in a nearly single-mode beam. The cryogenically-cooled Ho :YAG laser employed a simple two mirror resonator with a plane input coupler mirror with high reflectivity $(>99.5 \%)$ at the lasing wavelength $(\sim 2.1 \mu \mathrm{m})$ and high transmission (> 90\%) at the pump wavelength and a concave output coupler mirror with a transmission of $10 \%$ at the lasing wavelength. A $30 \mathrm{~mm}$ long Ho:YAG crytsal with 0.5 (at.) $\% \mathrm{Ho}^{3+}$ concentration and antireflection coated end faces was used as the gain medium. The latter was mounted in a copper heat-sink which was itself mounted in a custom designed liquid nitrogen dewar cryostat with two antireflection coated glass windows allowing operation at temperatures down to $\sim 80 \mathrm{~K}$. The mirrors forming the laser cavity were located outside the cryostat and pump light from the Tm fibre laser was focussed into the Ho:YAG crytsal using a lens arrangement also located outside the cryostat. Unfortunately, the windows used in this preliminary study were of rather poor quality and absorbed $\sim 8 \%$ of the intracavity laser power resulting in strong thermal effects. As a result, the pump powers used for our experiments were limited to $\sim 28 \mathrm{~W}$ yielding only $\sim 8 \mathrm{~W}$ of output at $\sim 2.1$ $\mu \mathrm{m}$. The lower power and efficiency than expected can be attributed to the poor quality windows. Even so, the performance was markedly improved over that at room temperature with a reduction in threshold pump power from $\sim 1.8 \mathrm{~W}$ (at room temperature) to $250 \mathrm{~mW}$ at liquid nitrogen temperature and an increase in output power by $\sim 2.3$ times compared to the output power at room temperature. We have also demonstrated operation of Ho:YAG at $1976 \mathrm{~nm}$ at multi-watt powers using a non-collinear pumping geometry corresponding to a quantum defect of only $2.3 \%$. With improved quality windows and by using volume Bragg gratings for the input mirror we expect to achieve a considerable improvement in performance at both wavelengths.

The latest results will be presented at the conference and the prospects for further power scaling will be discussed.

\section{References}

[1] D. C. Brown, "The promise of cryogenic solid-state lasers," IEEE J. Sel. Top. Quantum Electron. 11, 587-599 (2005).

[2] D. J. Ripin, J. R. Ochoa, R. L. Aggarwal, and T. Y. Fan, “165-W cryogenically cooled Yb:YAG laser,” Opt. Lett. 29, 2154 (2004). 